\title{
An Application of the Modified Reductive Perturbation Method to a Generalized Boussinesq Equation
}

\begin{abstract}
In this work, we apply "the modified reductive perturbation method" to the generalized Boussinesq equation and obtain various form of generalized $\mathrm{KdV}$ equations as the evolution equations. Seeking a localized travelling wave solutions for these evolution equations we determine the scale parameters $g_{1}$ and $g_{2}$, which corresponds to the correction terms in the wave speed, so as to remove the possible secularities that might occur. Depending on the sign and the values of certain parameters the resulting solutions are shown to be a solitary wave or a periodic solution. The suitability of the method is also shown by comparing the results with the exact travelling wave solution for the generalized Boussinesq equation.
\end{abstract}

Keywords: modified reductive perturbation method, generalized Boussinesq equation, generalized KdV equations

$\operatorname{PACS}^{\circledast}$ (2010). 47.35.Fg

Hilmi Demiray: Department of Mathematics, Faculty of Arts and Sciences, Isik University, Sile, 34980 Istanbul Turkey E-mail:demiray@isikun.edu.tr

\section{Introduction}

Due to nonlinearity of the governing equations in fluidfilled elastic tubes, in shallow water waves and in collisionless cold plasma, in expanding the field quantities into perturbation series for weakly dispersive case, the lowest order term in the perturbation expansion is governed by the Korteweg-de Vries equation (Davidson [1], Demiray [2]). The use of the classical reductive perturbation method in studying the higher order terms in the perturbation expansion leads to some secular terms in the solution. In order to eliminate such secularities Sugimoto and Kakutani [3] introduced some slow scale variables, Kodama and Taniuti [4] presented the re-normalization procedure. Another attempt to remove such secularities is made by Kraenkel et al. [5] for long water waves by use of the multiple time scale expansion.

In order to remove these uncertainties, Malfliet and Wieers [6] presented a dressed solitary wave approach, which is based on the assumption that the field variables admit localized travelling wave solution. Then, for the long wave limit, they expanded the field variables and the wave speed into a power series of the wave number, which is assumed to be the only smallness parameter, and obtained the explicit solution for various order terms in the expansion. However, this approach can only be used when one studies progressive wave solution to the original nonlinear equations and it does not give any idea about the form of evolution equations governing the various order terms in the perturbation expansion. In our previous paper [7], we have presented the modified reductive perturbation method to examine the contributions of higher order terms in the perturbation expansion and applied it to weakly dispersive ion-acoustic plasma waves and solitary waves in a fluid filled elastic tube [8]. In these works, we have shown that the lowest order term in the perturbation expansion is governed by the nonlinear Korteweg-de Vries equation, whereas the higher order terms in the expansion are governed by the degenerate (linearized) Korteweg-de Vries equation with nonhomogeneous term. By employing the hyperbolic tangent method a progressive wave solution was sought and the possible secularities were removed. The basic idea in this method was the inclusion of higher order dispersive effects through the introduction of the scaling parameter $g$, to balance the higher order nonlinearities with dispersion. The negligence of higher order dispersive effects in the classical reductive perturbation method leads to the imbalance between the nonlinearity and the dispersion, which resulted in some secular terms in the solution of evolution equations.

In the present work, we apply the modified reductive perturbation method to the generalized Boussinesq equation and obtained various form of generalized KdV equations as the evolution equations. Seeking a localized travelling wave solutions to these evolution equations we determined the scale parameters $g_{1}$ and $g_{2}$, which 
corresponds to the correction terms to the wave speed, so as to remove the possible secularities. Depending on the sign and the values of certain parameters the resulting solutions are shown to be a solitary wave or a periodic solution. The suitability of the method is also shown by comparing the result with the exact travelling wave solution of the generalized Boussinesq equation.

\section{Modified reductive perturbation formalism for the generalized Boussinesq equation}

The one dimensional form of the generalized Boussinesq equation is given by [9]

$$
u_{t t}-u_{x x}+u_{x x x x}-\frac{1}{n+1}\left(u^{n+1}\right)_{x x}=0 .
$$

where $n \geq 1$ is a positive integer and $u$ is the velocity in the $x$ direction. The dispersion relation of the linearized form of equation (1) may be given by

$$
\omega=k\left(1+k^{2}\right)^{1 / 2}
$$

where $\omega$ is the angular frequency and $k$ is the wave number. For our future purposes, it will be convenient to introduce the following stretched coordinates

$$
\xi=\varepsilon^{n / 2}(x-t), \quad \tau=\varepsilon^{3 n / 2} g t,
$$

where $\varepsilon=k^{2 / n}$ is the smallness parameter and $g$ is a scale parameter to be determined from the solution of the field equations. We shall further assume that the field variable $u$ and the scale parameter $g$ can be expanded into perturbation series in $\varepsilon$ as

$$
u=\sum_{l=0}^{\infty} \varepsilon^{n l+1} u_{l+1}(\xi, \tau), \quad g=1+\sum_{l=1}^{\infty} \varepsilon^{n l} g_{l},
$$

where the coefficient functions $u_{l}(\xi, \tau)$ and the constants $g_{l}$ are to be determined from the solution of the field equations. Introducing (3) and (4) in equation (1) and setting the coefficients of like powers of $\varepsilon$ equal to zero, the following sets of differential equations are obtained:

$O(\varepsilon)$ equation:

$$
\frac{\partial}{\partial \xi}\left[-2 \frac{\partial u_{1}}{\partial \tau}-\frac{1}{n+1} \frac{\partial}{\partial \xi}\left(u_{1}^{n+1}\right)+\frac{\partial^{3} u_{1}}{\partial \xi^{3}}\right]=0 .
$$

$O\left(\varepsilon^{n+1}\right)$ equation:

$$
-2 \frac{\partial^{2} u_{1}}{\partial \xi \partial \tau}-\frac{\partial^{2}}{\partial \xi^{2}}\left(u_{1}^{n} u_{2}\right)+\frac{\partial^{4} u_{2}}{\partial \xi^{4}}+\frac{\partial^{2} u_{1}}{\partial \tau^{2}}-2 g_{1} \frac{\partial^{2} u_{1}}{\partial \xi \partial \tau}=0
$$

$O\left(\varepsilon^{2 n+1}\right)$ equation:

$$
\begin{aligned}
& -2 \frac{\partial^{2} u_{3}}{\partial \xi \partial \tau}-2 g_{1} \frac{\partial^{2} u_{2}}{\partial \xi \partial \tau}-2 g_{2} \frac{\partial^{2} u_{1}}{\partial \xi \partial \tau}+\frac{\partial^{2} u_{2}}{\partial \tau^{2}}+2 g_{1} \frac{\partial^{2} u_{1}}{\partial \tau^{2}} \\
& -\frac{1}{n+1} \frac{\partial^{2}}{\xi^{2}}\left[\left(\begin{array}{c}
n+1 \\
2
\end{array}\right) u_{1}^{n-1} u_{2}^{2}+\left(\begin{array}{c}
n+1 \\
1
\end{array}\right) u_{1}^{n} u_{3}\right]+\frac{\partial^{4} u_{3}}{\partial \xi^{4}}=0 .
\end{aligned}
$$

where $\left(\begin{array}{l}n \\ m\end{array}\right)$ is the Binomial coefficient.

\subsection{Solution of the field equations}

Integrating (5) with respect to $\xi$ one gets

$$
\frac{\partial u_{1}}{\partial \tau}+\frac{1}{2} u_{1}^{n} \frac{\partial u_{1}}{\partial \xi}-\frac{1}{2} \frac{\partial^{3} u_{1}}{\partial \xi^{3}}=f(\tau)
$$

where $f(\tau)$ is an arbitrary function of its argument and can be chosen to be zero. Then, the evolution equation becomes

$$
\frac{\partial u_{1}}{\partial \tau}+\frac{1}{2} u_{1}^{n} \frac{\partial u_{1}}{\partial \xi}-\frac{1}{2} \frac{\partial^{3} u_{1}}{\partial \xi^{3}}=0
$$

Here, the equation (9) is known as the generalized Korteweg-de Vries equation.

Inserting (9) into (6), the $O\left(\varepsilon^{n+1}\right)$ equation takes the following form

$$
\begin{gathered}
\frac{\partial}{\partial \xi}\left[-2 \frac{\partial u_{2}}{\partial \tau}-\frac{\partial}{\partial \xi}\left(u_{1}^{n} u_{2}\right)+\frac{\partial^{3} u_{2}}{\partial \xi^{3}}-\frac{1}{2(n+1)} \frac{\partial}{\partial \tau}\left(u_{1}^{n+1}\right)\right. \\
\left.+\frac{1}{2} \frac{\partial^{3} u_{1}}{\partial \xi^{2} \partial \tau}-2 g_{1} \frac{\partial u_{1}}{\partial \tau}\right]=0 .
\end{gathered}
$$

Integrating the equation (10) with respect to $\xi$ and setting the resulting arbitrary function equal to zero the following equation is obtained

$$
\frac{\partial u_{2}}{\partial \tau}+\frac{1}{2} \frac{\partial}{\partial \xi}\left(u_{1}^{n} u_{2}\right)-\frac{1}{2} \frac{\partial^{3} u_{2}}{\partial \xi^{3}}=S_{1}\left(u_{1}\right)
$$

where $S_{1}\left(u_{1}\right)$ is defined by

$$
S_{1}\left(u_{1}\right)=-g_{1} \frac{\partial u_{1}}{\partial \tau}+\frac{1}{4} \frac{\partial^{3} u_{1}}{\partial \xi^{2} \partial \tau}-\frac{1}{4(n+1)} \frac{\partial}{\partial \tau}\left(u_{1}^{n+1}\right) .
$$


Here the equation (11) is the degenerate form of the generalized Korteweg-de Vries equation with a nonhomogeneous term.

Finally, to obtain the solution for $O\left(\varepsilon^{2 n+1}\right)$ equation we introduce the equations (9) and (11) into (7) and obtain the following evolution equation

$$
\frac{\partial u_{3}}{\partial \tau}+\frac{1}{2} \frac{\partial}{\partial \xi}\left(u_{1}^{n} u_{3}\right)-\frac{1}{2} \frac{\partial^{3} u_{3}}{\partial \xi^{3}}=S_{2}\left(u_{1}, u_{2}\right)
$$

where $S_{2}\left(u_{1}, u_{2}\right)$ is defined by

$$
\frac{\partial S_{2}}{\partial \xi}=-g_{1} \frac{\partial^{2} u_{2}}{\partial \xi \partial \tau}-g_{2} \frac{\partial^{2} u_{1}}{\partial \xi \partial \tau}+\frac{1}{2} \frac{\partial^{2} u_{2}}{\partial \tau^{2}}+g_{1} \frac{\partial^{2} u_{1}}{\partial \tau^{2}}-\frac{n}{4} \frac{\partial^{2}}{\partial \xi^{2}}\left(u_{1}^{n-1} u_{2}^{2}\right) .
$$

Again, the equation (13) is the degenerate form of the generalized KdV equation and it is linear in $u_{3}$ and contains the inhomogeneous term $S_{2}\left(u_{1}, u_{2}\right)$. Here, one should note that $g_{1}$ and $g_{2}$ remain as some unknown constants and they should be determined from the removal of some secular term that might occur in the travelling wave solution.

\subsection{Progressive wave solution}

In this sub-section we shall give a progressive wave solution to the evolution equations described in (9), (11) and (13). To this aim, the following type of solution is proposed

$$
u_{i}=U_{i}(\zeta), \quad \zeta=\alpha(\xi-v \tau),(i=1,2,3)
$$

where $\alpha$ and $v$ are two constants to be determined from the solution. Substituting (15) into (9) yields

$$
-v U_{1}^{\prime}+\frac{1}{2} U_{1}^{n} U_{1}^{\prime}-\frac{1}{2} \alpha^{2} U_{1}^{\prime \prime \prime}=0
$$

Here a prime denotes the differentiation of the corresponding quantity with respect to $\zeta$. By integrating (16) with respect to $\zeta$ and employing the localization condition i.e., $U_{1}$ and its various order derivatives vanish as $\zeta \rightarrow \pm \infty$, one gets

$$
-v U_{1}+\frac{1}{2(n+1)} U_{1}^{n+1}-\frac{1}{2} \alpha^{2} U_{1}^{\prime \prime}=0 .
$$

This nonlinear differential equation admits the solution of the form

$$
U_{1}=a \operatorname{sech}^{2 / n} \zeta
$$

where $a$ is the wave amplitude. Inserting (18) into equation (17) and setting the coefficients of like powers of sech $\zeta$ equal to zero one obtains

$$
\alpha^{2}+\frac{a^{n} n^{2}}{2(n+1)(n+2)}=0, \quad v+2 \frac{\alpha^{2}}{n^{2}}=0
$$

As is seen from equation (19), depending on the sign of $a$ and the value of $n, \alpha$ becomes real or purely imaginary. We shall study the following cases separately: (i) $a<0$ and $n$ is an odd integer, (ii) $n$ is an even integer.

(i) For this case setting $a=-b(b>0)$ and $n=2 m+1$, where $m$ is a positive integer, from equation (19) one has

$$
\alpha^{2}=\frac{(2 m+1)^{2} b^{2 m+1}}{4(m+1)(2 m+3)}, \quad v=-\frac{2 \alpha^{2}}{(2 m+1)^{2}},
$$

and the solution for $U_{1}$ becomes

$$
U_{1}=-b \operatorname{sech}^{2 /(2 m+1)} \zeta .
$$

The solution (21) is a typical solitary wave.

Introducing (15) into equation (11) one has

$$
\begin{aligned}
-v U_{2}^{\prime} & +\frac{1}{2}\left(U_{1}^{2 m+1} U_{2}\right)^{\prime}-\frac{1}{2} \alpha^{2} U_{2}^{\prime \prime \prime} \\
= & g_{1} v U_{1}^{\prime}-\frac{\alpha^{2}}{4} v U_{1}^{\prime \prime \prime}+\frac{v}{8(m+1)}\left(U_{1}^{2 m+2}\right)^{\prime} .
\end{aligned}
$$

Integrating (22) with respect to $\zeta$ utilizing the localization condition and inserting the solution (20) and (21) we have

$$
\begin{aligned}
U_{2}^{\prime \prime} & +\frac{4}{(2 m+1)^{2}}\left[(m+1)(2 m+3) \operatorname{sech}^{2} \zeta-1\right] U_{2} \\
& =\frac{4 b}{(2 m+1)^{2}}\left[-g_{1}+\frac{b^{2 m+1}}{4(m+1)(2 m+3)}\right] \operatorname{sech}^{2 /(2 m+1)} \zeta .
\end{aligned}
$$

Here, the term on the right side causes to the secularity in the solution. Thus, the coefficient of $\operatorname{sech}^{2 /(2 m+1)} \zeta$ must vanish, which yields

$$
g_{1}=\frac{\alpha^{2}}{(2 m+1)^{2}}
$$

This gives the first correction term to the wave speed. Thus, the particular solution for $U_{2}$ will be zero. Here, it is to be noted that, without loosing the generality of the problem we may take $U_{2}=0$ and $S_{1}\left(U_{1}\right)=0$. 
To obtain the particular solution for the evolution equation (13) we set $U_{2}=0$ and it reduces to

$$
-v U_{3}^{\prime}+\frac{1}{2}\left(U_{1}^{2 m+1} U_{3}\right)^{\prime}-\frac{1}{2} \alpha^{2} U_{3}^{\prime \prime \prime}=\left(g_{2} v+g_{1} v^{2}\right) U_{1}^{\prime} .
$$

Integrating (25) with respect $\zeta$ and utilizing the localization condition we obtain

$$
-v U_{3}+\frac{1}{2}\left(U_{1}^{2 m+1} U_{3}\right)-\frac{\alpha^{2}}{2} U_{3}^{\prime \prime}=-b v\left(g_{2}+v g_{1}\right) \operatorname{sech}^{2 /(2 m+1)} \zeta
$$

Here, again, in order to remove the secularity the coefficient of $\operatorname{sech}^{2 /(2 m+1)} \zeta$ must vanish; which results in

$$
g_{2}+v g_{1}=0, \quad \text { or } \quad g_{2}=\frac{2 \alpha^{4}}{(2 m+1)^{4}}
$$

Here, $g_{2}$ is the second correction term to the wave speed and the particular solution for $U_{3}$ may be taken to be zero. Thus, the solution takes the following form

$$
u=-\varepsilon b \operatorname{sech}^{2 /(2 m+1)} \zeta
$$

with

$$
\zeta=\alpha \varepsilon^{(2 m+2) / 2}\left\{x-t+\varepsilon^{2 m+1} \frac{2 \alpha^{2}}{(2 m+1)^{2}} t+\varepsilon^{4 m+2} \frac{2 \alpha^{4}}{(2 m+1)^{4}} t+\cdots\right\} .
$$

If one sets $m=0(n=1)$ in equations (20)-(29) the result will be exactly the same with that of [11].

(ii) The case of $n$ is an even positive integer

In this case $n$ can be expressed as $n=2 m$, where $m$ is a positive integer. Then, from equation (19), it is seen that $\alpha$ is purely imaginary number with the expression

$$
\alpha=i \beta, \quad \beta=\frac{a^{m} m}{[(m+1)(2 m+1)]^{1 / 2}}, \quad v=\frac{\beta^{2}}{2 m^{2}} .
$$

Then, the progressive wave solution of the first order term in the perturbation expansion reduces to

$$
U_{1}=a \sec ^{1 / m} \zeta, \quad \zeta=\beta(\xi-v \tau), \quad|\zeta|<\frac{\pi}{2} .
$$

The solution defined on the finite domain $|\zeta|<\frac{\pi}{2}$ is known as the periodic solution.
Introducing (31) into equation (11) one obtains

$$
\begin{aligned}
U_{2}^{\prime \prime} & +\frac{1}{m^{2}}\left[(m+1)(2 m+1) \sec ^{2} \zeta-1\right] U_{2} \\
& =-\frac{1}{m^{2}}\left(g_{1}+\frac{\beta^{2}}{4 m^{2}}\right) a \sec ^{1 / m} \zeta .
\end{aligned}
$$

The term on the right hand side of (32) causes to the secularity. Thus, the coefficient of $\sec ^{1 / m} \zeta$ must vanish

$$
g_{1}+\frac{\beta^{2}}{4 m^{2}}=0, \quad \text { or } \quad g_{1}=-\frac{\beta^{2}}{4 m^{2}}
$$

The particular solution of equation (32) may be taken to be zero, i.e., $U_{2}=0$. In this case, the evolution (13) takes the following form

$$
\begin{aligned}
U_{3}^{\prime \prime}+ & \frac{1}{m^{2}}\left[(m+1)(2 m+1) \sec ^{2} \zeta-1\right] U_{3} \\
& =-\beta a\left[g_{2}-\frac{\beta^{4}}{8 m^{4}}\right] \sec ^{1 / m} \zeta .
\end{aligned}
$$

In order to remove the secularity in the solution on must have

$$
g_{2}-\frac{\beta^{4}}{8 m^{4}}=0, \text { or } \quad g_{2}=\frac{\beta^{4}}{8 m^{4}}
$$

Thus, the total solution up to $O\left(\varepsilon^{2 n+1}\right)$ takes the following form

$$
u=\varepsilon a \sec ^{1 / m} \zeta, \quad \zeta=\varepsilon^{m} \beta\left[x-t-\varepsilon^{2 m} \frac{\beta^{2}}{2 m^{2}} t+\varepsilon^{4 m} \frac{\beta^{4}}{8 m^{4}} t-\cdots\right] .
$$

\subsection{Comparison of the result with exact solution}

In this sub-section we shall compare the result obtained here through the use of modified reductive perturbation method with the exact solution of generalized Boussinesq equation. For that, we shall seek a progressive wave solution to the equation (1) of the form

$$
U=a \operatorname{sech}^{1 / n} \zeta, \quad \zeta=\gamma(x-c t),
$$

provided that the following relations hold true

$$
\gamma^{2}+\frac{a^{n} n^{2}}{2(n+1)(n+2)}=0, \quad c^{2}-1+4 \frac{\gamma^{2}}{n^{2}}=0 .
$$


The parameter $\gamma$ will be a real number when $a=-d,(d>0)$ and $n$ is an odd integer, i.e. $n=2 m+1$, the equation (38) becomes

$$
\gamma^{2}=\frac{(2 m+1)^{2} d^{2 m+1}}{4(m+1)(2 m+3)}, \quad c^{2}-1+4 \frac{\gamma^{2}}{(2 m+1)^{2}}=0
$$

In this case the solution reads

$$
U=-d \operatorname{sech}^{2 /(2 m+1)} \zeta, \quad \zeta=\gamma(x-c t) .
$$

Setting $d=\varepsilon b$, where the constant $b$ is the same as in (21), the solution becomes

$$
U=-\varepsilon b \operatorname{sech}^{2 /(2 m+1)} \zeta, \quad \gamma^{2}=\varepsilon^{2 m+1} \alpha^{2}
$$

Here $\alpha$ is the same as in (23) and $\zeta$ takes the following form

$$
\zeta=\varepsilon^{m+1 / 2} \alpha\left[x-t \sqrt{1-\varepsilon^{2 m+1} \frac{4 \alpha^{2}}{(2 m+1)^{2}}}\right]
$$

Expanding (42) into a power series of the smallness parameter $\varepsilon$ we obtain

$$
\begin{aligned}
\zeta=\varepsilon^{m+1 / 2} \alpha[ & x-t+\varepsilon^{2 m+1} \frac{2 \alpha^{2}}{(2 m+1)^{2}} t+\varepsilon^{4 m+2} \frac{2 \alpha^{4}}{(2 m+1)^{4}} t \\
& \left.+\varepsilon^{6 m+3} \frac{4 \alpha^{6}}{(2 m+1)^{6}}+\cdots\right] .
\end{aligned}
$$

This solution is exactly the same with that of given in (29).

When $n$ is an even integer, say $n=2 m, \gamma$ becomes purely imaginary number and the solution takes the following form

$$
U=f \sec ^{1 / m} \zeta, \quad \zeta=\Gamma(x-c t)
$$

with

$$
\Gamma^{2}=\frac{m^{2} f^{2 m}}{(m+1)(2 m+3)}, \quad c=\sqrt{1+\frac{\Gamma^{2}}{m^{2}}} .
$$

where $f$ is the constant amplitude.

Setting $f=\varepsilon a$, where the constant $a$ is the same as in equation (36), the solution becomes

$$
U=\varepsilon a \sec ^{1 / m} \zeta, \quad \zeta=\varepsilon^{m} \beta(x-c t), \quad c=\sqrt{1+\varepsilon^{2 m} \frac{\beta^{2}}{m^{2}}} .
$$

Here $\beta$ is the same as defined in (30). Expanding $c$ into a power series of the smallness parameter $\varepsilon$ one obtains

$$
\zeta=\varepsilon^{m} \beta\left[x-t-\varepsilon^{2 m} \frac{\beta^{2}}{2 m^{2}} t+\varepsilon^{4 m} \frac{\beta^{4}}{8 m^{4}} t-\cdots\right]
$$

This result is exactly the same with that of given in (36).

\section{Concluding remarks}

The modified reductive method is applied to the generalized Boussinesq equation (1) to determine the higher order correction terms by assuming that the solution is free from secular terms.

Received: August 26, 2011. Accepted: September 24, 2012.

\section{References}

[1] N. Davidson, Methods in Nonlinear Plasma Theory, Academic Press, New York, 1972.

[2] H. Demiray, Solitary waves in prestressed elastic tubes, Bulletin of Mathematical Biology, 58(1991), 939-955.

[3] N. Sugimoto and T. Kakutani, Note on higher order terms in reductive perturbation theory, J. Phys. Soc. Japan, 43(1977), 1469-1470.

[4] Y. Kodama and T. Taniuti, Higher order approximation in reductive perturbation method: 1 . Weakly dispersive system, J. Phys. Soc. Japan, 45(1978), 298-310.

[5] R. A. Kraenkel, M. A. Manna and J. G. Pereira, The Korteweg-de Vries hierarchy and long water waves, J. Math. Phys., 36(1995), 307-320.

[6] M. Malfliet and E. Wieers, The theory of nonlinear ion-acoustic waves revisited, J. Plasma Phys., 56(1996), 441-453.

[7] H. Demiray, A modified reductive perturbation method as applied to nonlinear ion-acoustic waves, J. Phys. Soc. Japan, 68(1999), 1833-1837.

[8] H. Demiray, On the contribution of higher order terms to solitary waves in fluid-filled elastic tubes, Z. Agnew. Math. Phys. (ZAMP), 51(2000), 75-91.

[9] Y. Liu, Instability of solitary waves for generalized Boussinesq equation, J. Dynamics and Differential Equations, 5(1993) 537-558.

[10] H. Demiray, A travelling wave solution to the KdV-Burgers equation, Applied Mathematics and Computation, 154(2004), 665-670.

[11] H. Demiray, The modified reductive perturbation method as applied to the Boussinesq equation, Z. Naturforsch., 62a(2007) 347-352. 